\title{
Iron Catalyzed Synthesis and Chemical Recycling of Telechelic, 1,3- Enchained Oligocyclobutanes
}

\author{
Megan Mohadjer Beromi ${ }^{1}$, C. Rose Kennedy ${ }^{1}$, Jarod M. Younker ${ }^{2}$, Alex E. Carpenter ${ }^{2}$, Sarah J. Mattler ${ }^{2}$, \\ Joseph A. Throckmorton ${ }^{2}$, and Paul J. Chirik ${ }^{1 *}$ \\ 1. Department of Chemistry, Princeton University, Princeton, New Jersey 08544, USA \\ 2. ExxonMobil Chemical, Baytown, TX, 77520, USA \\ To whom correspondence should be addressed: Email: pchirik@princeton.edu
}

Closed-loop recycling offers the opportunity to help mitigate plastic waste through reversible polymer construction and deconstruction. While examples of the chemical recycling polymers are known, few have been applied to materials derived from abundant commodity olefinic monomers that are the building blocks of ubiquitous plastic resins. Here we describe a [2+2] cycloaddition oligomerization of 1,3-butadiene to yield a previously unrealized telechelic microstructure of $\left(1, n^{\prime}\right.$-divinyl)oligocyclobutane. This material is thermally stable, has stereoregular segments arising from chain-end control, and exhibits high crystallinity even at low molecular weight. Exposure of the oligocyclobutane to vacuum in the presence of the pyridine(diimine) iron precatalyst used to synthesize it resulted in deoligomerization to generate pristine butadiene, demonstrating a rare example of closed-loop chemical recycling of an oligomeric material derived from a commodity hydrocarbon feedstock.

Single-use plastics have become ubiquitous in consumer and industrial products that enable conveniences of modern life due to their low cost, lightweight, durability, and high stability. However, these features also present a challenge for waste management, resulting in the environmental accumulation of end-of-life plastics ${ }^{1,2,3}$. With current recycling rates of approximately $10 \%$, the fate of the majority of commodity plastics are either in landfills or the environment ${ }^{2,4}$. Additionally, current mechanical recycling practices incur substantial loss-of-value in each cycle producing materials with inferior physical properties ${ }^{2}$.

Chemical recycling offers an alternative strategy and holds promise for up-cycling waste plastics to their constituent monomers or other value-added products ${ }^{2,5,6,7}$. Recent efforts toward chemical recycling have focused on the introduction of new polymeric structures bonded through covalent, heteroatomic linkages that are susceptible to reversible chemical cleavage (Figure 1A) ${ }^{8,9,10,11,12,13,14}$. While these examples provide valuable proof-of-concept, state-of-the-art methods still require specialized monomers that are dwarfed in scale by those used for commodity hydrocarbon resins (Figure 1B) ${ }^{15}$. Further, the attendant properties (i.e., crystallinity, glass transition temperature, stress/strain, chemical inertness, tear, gas-barrier) of these polymers often exhibit attributes that make them unsuitable for commercial application. By contrast, the six most common plastic resins produced industrially exhibit excellent material properties and are derived from monomers obtained directly from abundant petrochemical feedstocks (e.g. ethylene, propylene, styrene, xylene); five are generated exclusively from olefinic monomers. ${ }^{16,17}$. As a consequence, there is a need to develop chemically recyclable polymers derived from hydrocarbon feedstocks that do not require the use of specialized monomers ${ }^{15,18,19}$. Further, these materials need to exhibit physical properties 
comparable to conventional polyolefins. In this regard, chemically recyclable semi-crystalline polymers are particularly rare. Here we describe the realization of this goal with the synthesis, characterization, and controlled deconstruction of oligomers consisting of 1,3-linked cyclobutane repeat units (Figure 1C). These findings have identified a fundamentally unique microstructure of polybutadiene amenable to closed-loop chemical recycling.

Results and discussion. It is well-established that pyridine(diimine)-supported iron complexes promote the intermolecular [2+2]-cycloaddition between alkenes, or alkenes and dienes ${ }^{20,21}$. Mechanistic studies support metallacyclic intermediates arising from oxidative cyclization of the unsaturated hydrocarbon substrates, often giving rise to cyclobutanes with high regio- and stereoselectivities ${ }^{20,21,22,23}$. For dienealkene [2+2] cycloaddition, the iron catalyst engages only one of the alkenes of the diene, suggesting that a cascade cycloaddition oligomerization of 1,3-butadiene to yield a new microstructure of oligobutadiene

\section{A. State-of-the-Art Reversible Polymerization Requires Specialty Monomers:}

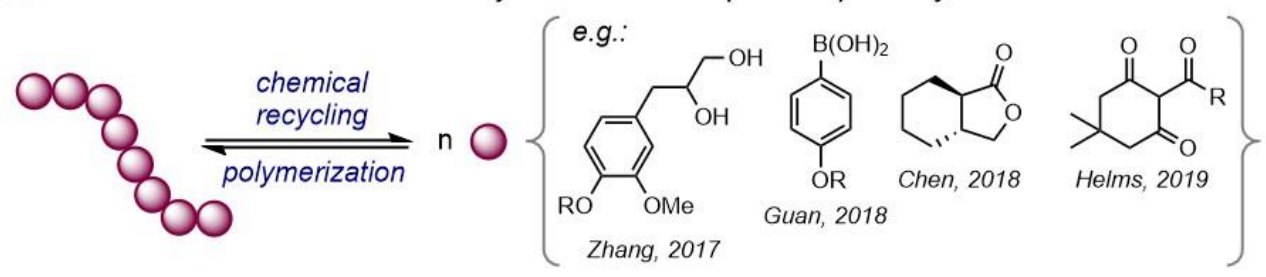

B. Five of the Top Six Commodity Plastic Resins are Olefin Derived:

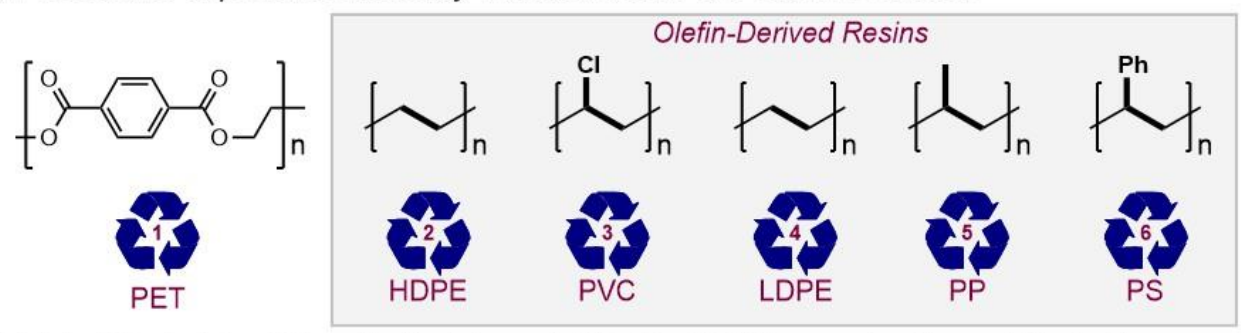

C. This Work: The Chemically Recyclable [2+2] Oligomerization of Butadiene:

Figure 1. Approaches to the closed-loop recycling of polymers. A. Current chemically reversible polymers utilize covalent linkages formed from tailored monomers (ref. 13-16). B. Five of the most common plastic resins are constructed from feedstock olefins and consist of chemically recalcitrant linkages. C. Accessing a chemically recyclable, olefin derived oligomer through the [2+2] oligomerization of butadiene. 
would be possible (Figure 2A). Dissolving the iron dinitrogen precatalyst, $\left(\left({ }^{\mathrm{Me}} \mathrm{PDI}\right) \mathrm{Fe} \mathrm{N}_{2}\right)_{2}\left(\mu-\mathrm{N}_{2}\right)\left({ }^{\mathrm{Me}} \mathrm{PDI}=\right.$ 2,6-(2,6-Me- $\left.\left.\mathrm{C}_{6} \mathrm{H}_{3}-\mathrm{N}=\mathrm{CMe}\right)_{2} \mathrm{C}_{5} \mathrm{H}_{3} \mathrm{~N}\right)$ in neat butadiene resulted in consumption of the liquid monomer after three days at $50{ }^{\circ} \mathrm{C}$ and generated a white solid. Removal of the volatiles and extraction of the residue with ethyl acetate yielded a soluble, semisolid fraction and an insoluble, hard, crystalline, light tan powder (Figure 2B).

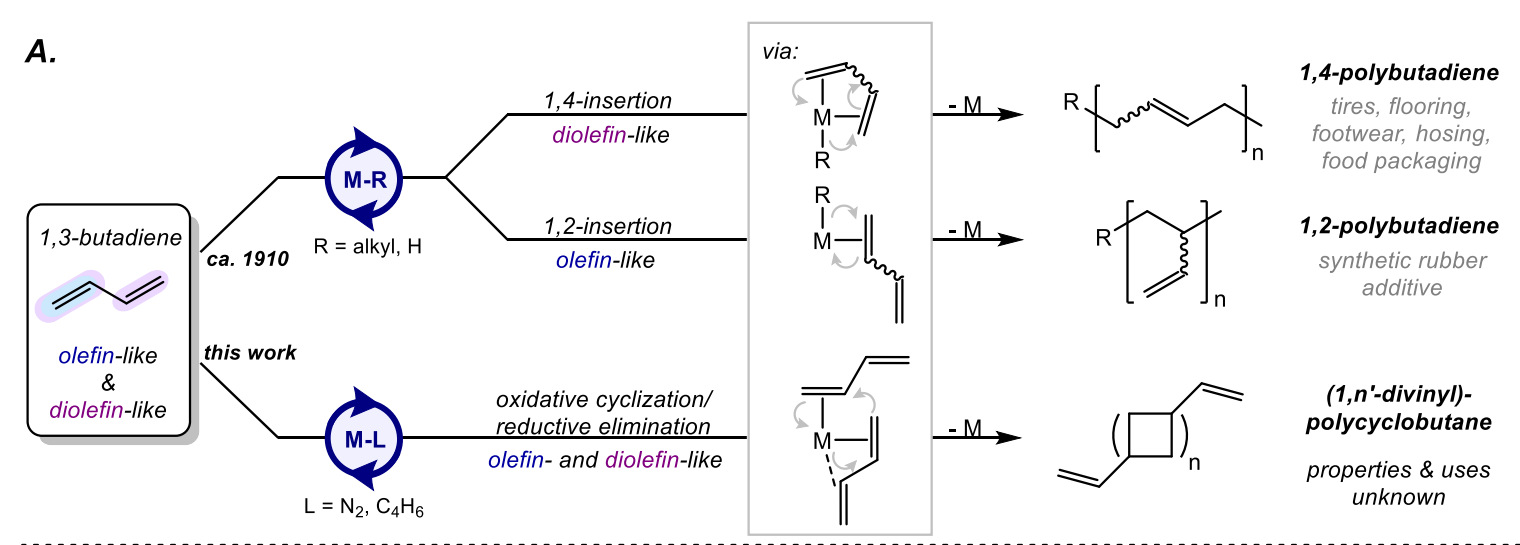

B.

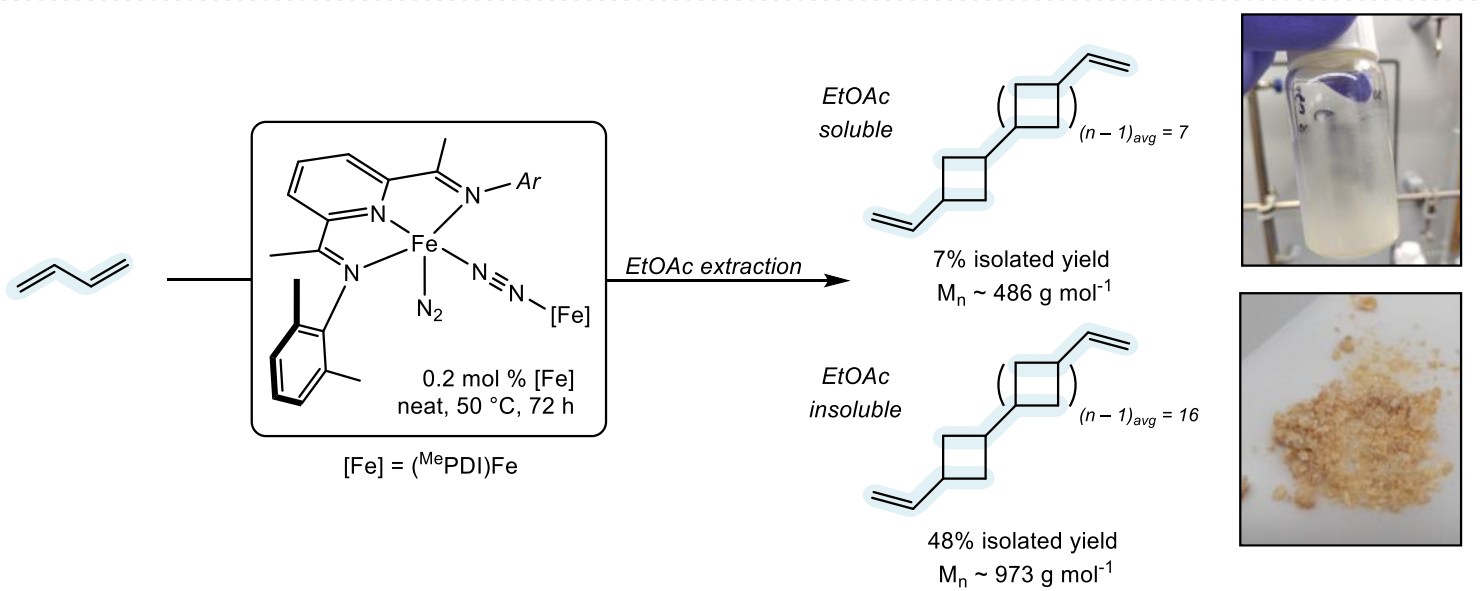

Figure 2. A unique microstructure of polybutadiene obtained through iron-catalyzed [2+2]cycloaddition/oligomerization. A. Known butadiene polymers have been obtained through insertion mechanisms, whereas polycyclobutane can be obtained from cascade oxidative cyclization/oligomerization. B. Oligomerization of butadiene under elevated temperatures and neat conditions produces lower molecular weight, organic soluble (1,n'-divinyl)polycyclobutane and higher molecular weight, insoluble (1,n'divinyl)polycyclobutane.

Characterization of the organic soluble fraction by 1D and 2D NMR spectroscopies established that the oligomer microstructure was distinct from that of known polymers resulting from 1,4- or 1,2-addition of butadiene (Figure 3). Instead, the spectroscopic data establish an oligomer of 1,3-linked cyclobutanes terminated by vinyl groups on both chain ends. NMR spectroscopic analysis of the crystalline fraction in 1,1,2,2-tetrachloroethane- $d_{2}$ at $120{ }^{\circ} \mathrm{C}$ revealed analogous features, differing only in the increased ratio of repeat units to chain ends (Figure S1A and Figure S2A). DFT (Density Functional Theory) simulation of 


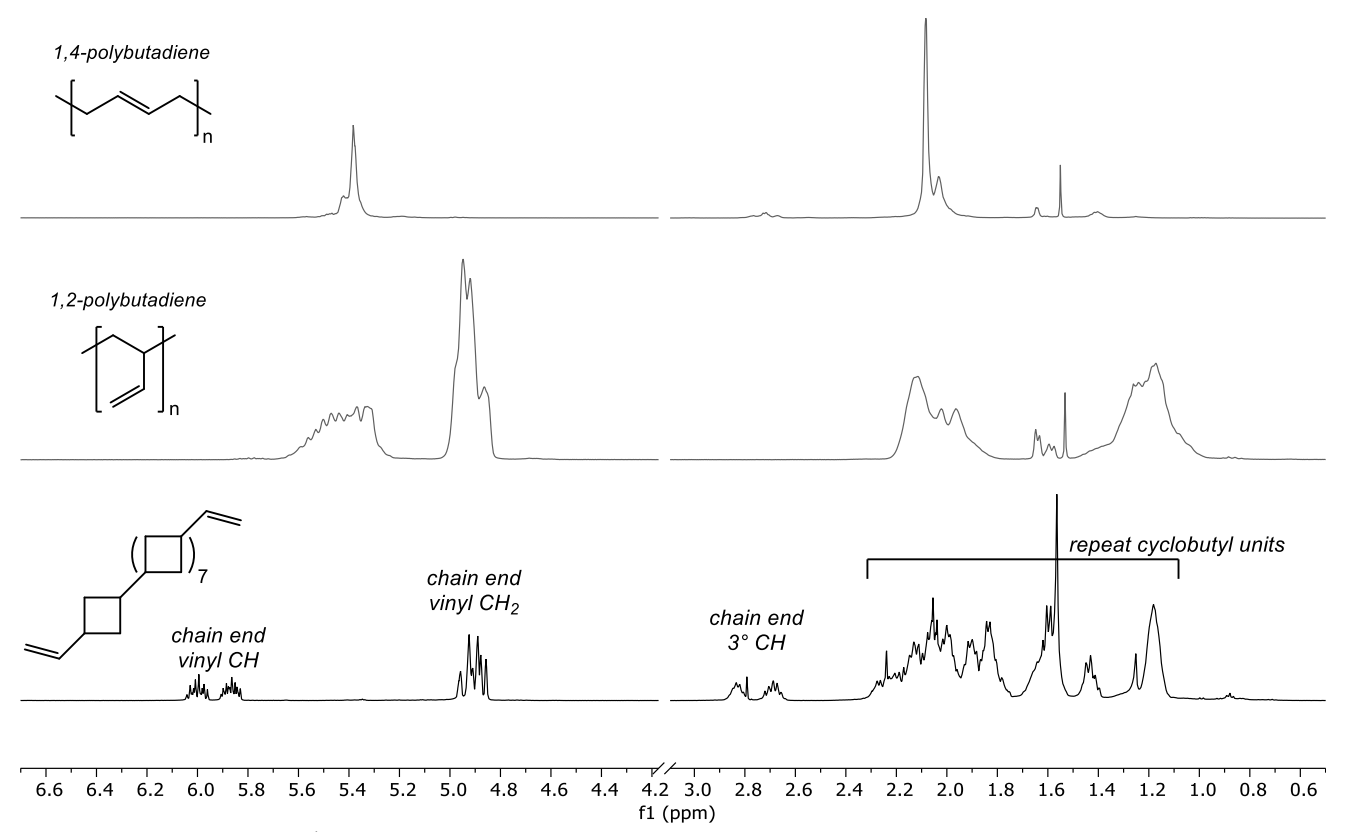

Figure 3. Comparison of the ${ }^{1} \mathrm{H}$ NMR spectra of polybutadiene microstructures. NMR spectra were obtained in $\mathrm{CDCl}_{3}$ at ambient probe temperature at either 400 or $500 \mathrm{MHz}$. Partial assignment of the polycyclobutane microstructure is shown; more detailed assignments are reported in the Supporting Information.

the NMR chemical shifts corroborated a 1,3-linked cyclobutane polymer structure. Quantitation of oligomer molecular weight, based on ${ }^{1} \mathrm{H}$ NMR integrations, revealed that the organic soluble material has a $\mathrm{M}_{\mathrm{n}}$ of $486 \mathrm{~g} \mathrm{~mol}^{-1}$, corresponding to an average of eight cyclobutyl repeat units, while the insoluble material is $973 \mathrm{~g} \mathrm{~mol}^{-1}$, or a number average chain of seventeen cyclobutyl rings. While both of these molecular weights were too low to be analyzed by gel permeation chromatography (GPC), the mass values obtained by ${ }^{1} \mathrm{H}$ NMR spectroscopy were corroborated by mass spectrometry (APCI MS) (Figure S4).

The iron-catalyzed [2+2] oligomerization of butadiene was also initiated in the presence of ethylene. Adding equimolar amounts of ethylene and 1,3-butadiene to a vessel containing $\left(\left({ }^{\mathrm{Me}} \mathrm{PDI}\right) \mathrm{FeN}_{2}\right)_{2}\left(\mu-\mathrm{N}_{2}\right)$ at ambient temperature over two days yielded vinylcyclobutane, as previously reported ${ }^{20}$, along with oligomeric products arising from further [2+2] cycloaddition/oligomerization of the formed vinylcyclobutane with butadiene. The resulting oligomers consisted of cyclobutane repeat units terminated by a vinyl group on only one end on the chain (Figure S5 and S6). This result indicates that tailored control of the chain end component of the oligocyclobutane is possible through choice of olefin coupling partner, representing a marked deviation from previous reports of 1,3-linked polycyclobutane structures ${ }^{24,25,26}$.

The unique structure of the oligocyclobutanes prompted more detailed characterization studies. While the products of butadiene [2+2]-cycloaddition/oligomerization are intrinsically achiral (meso), each cyclobutane repeat unit may be constituted with 1,3-substituents in either a syn or anti disposition. High field NMR spectroscopy enabled the assignment and quantification of chain end diad sequences and 
polymer chain triad sequences. The results of comprehensive, quantitative NMR peak identification and integration yield the following statistics on sequence distribution. First, there are equal amounts of syn and anti cyclobutane rings; the same is true at the vinyl chain ends. Second, adjacent cyclobutane rings have a higher probability ( $60 \%$ ) of having the opposite disposition (i.e., anti-syn or syn-anti). Density functional and transition state theory (DFT/TST) calculations for [2+2]-cycloaddition of butadiene by [( $\left.\left.{ }^{\mathrm{Me} P D I}\right) \mathrm{Fe}\right]$ agree with the NMR analysis, namely, the vinyl chain end cyclobutane has equal probability of being anti or $\operatorname{syn}\left(\Delta \Delta \mathrm{G}^{\ddagger}<1 \mathrm{kcal} / \mathrm{mol}\right.$ for reductive elimination, see Figure S17), and subsequent [2+2]-cycloadditions favor the opposite disposition $\left(\Delta \Delta \mathrm{G}^{\ddagger} \sim 4 \mathrm{kcal} / \mathrm{mol}\right.$ for reductive elimination, see Figure $\left.\mathrm{S} 17\right)$. Further, while alternative pyridine(diimine) iron precatalysts were also found to be competent for the [2+2]-cycloaddition polymerization, they afforded oligomers of similar or lower molecular weight and no perturbation of the previously observed tacticity (Table S5).

The crystallinity of the insoluble fraction obtained from butadiene homo-oligomerization was confirmed by powder X-ray diffraction studies. While unambiguous structural determination was precluded by the inability to obtain a crystal suitable for single crystal X-ray diffraction, comparison of the powder pattern to that of known hydrocarbon polymers bore no analogy, confirming a unique crystalline microstructure. Variable-temperature Wide Angle X-ray Scattering (VT-WAXS) revealed that the oligomer regained crystallinity upon cooling from $170{ }^{\circ} \mathrm{C}$ to $30{ }^{\circ} \mathrm{C}$ (Figure 4A and Table S6), with a rotator phase appearing at a temperature range of $110-130{ }^{\circ} \mathrm{C}$. Taken together, these data suggest a temperature-dependent ordering of domains within the oligo-cyclobutane structure.

Thermal data obtained on the crystalline oligomer lends insights into the robustness of this new microstructure. Thermal Gravimetric Analysis (TGA) of the crystalline material revealed a bulk decomposition event at an onset temperature of $413{ }^{\circ} \mathrm{C}$ (Figure S12). Analysis of the volatile decomposition products by TGA-GC/MS indicates that butadiene is not evolved during bulk decomposition; thus, retro-
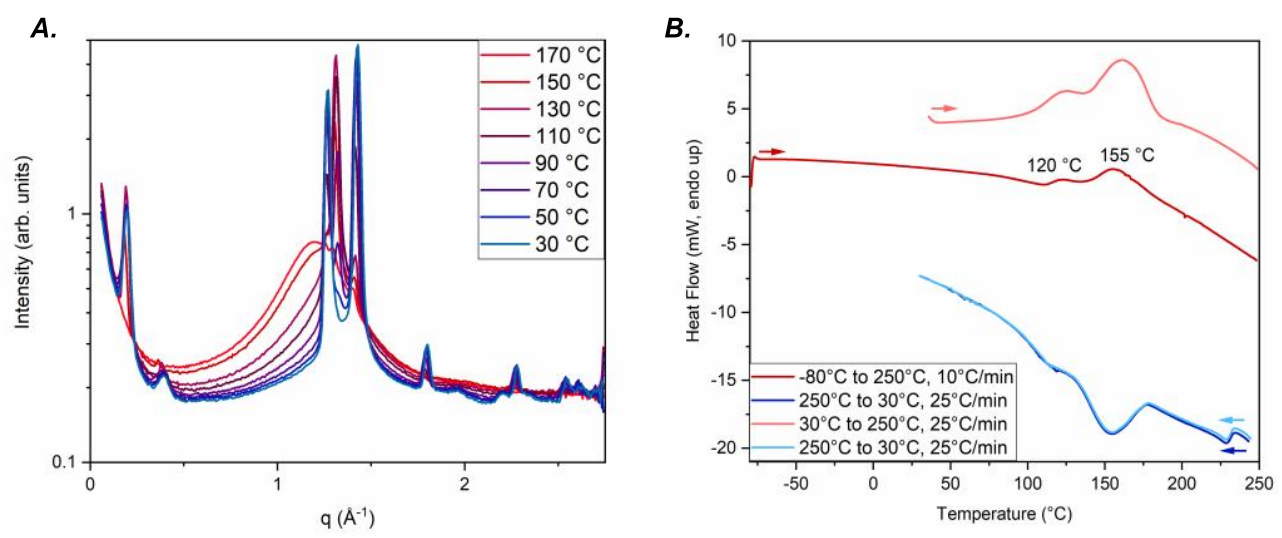

Figure 4. Select thermal data for crystalline (1,n'-divinyl)polycyclobutane. A. Variable temperature WAXS data indicating amorphous material crystallizing upon cooling. B. DSC data demonstrating retention of melting and crystallization temperatures upon temperature cycling. Isothermal regions have been omitted for clarity; the full data set can be found in Figure S13. 
cyclization is not thermally induced. The overall thermal properties of the material parallels that of 1,4polybutadiene of approximately the same $M_{n}$, despite the drastically different morphologies of the materials. Differential Scanning Calorimetry (DSC) indicated that the crystalline domains of the cyclobutyl oligomer are recoverable upon heating from $-80{ }^{\circ} \mathrm{C}$ to $250{ }^{\circ} \mathrm{C}$ and subsequently cycling from $250{ }^{\circ} \mathrm{C}$ to 30 ${ }^{\circ} \mathrm{C}$ twice (Figure 4B). The bimodal melting temperatures are ascribed to an initial transition of the crystalline domain to a rotator phase (onset at $\sim 110{ }^{\circ} \mathrm{C}$ ), with subsequent transition to completely amorphous material (onset at $135^{\circ} \mathrm{C}$ ), corroborating the VT-WAXS features. The melting event at $155^{\circ} \mathrm{C}$ is analogous to the reported melting temperature of the $90 \%$ trans 1,4-polybutadiene congener ${ }^{27}$. Additional DSC analysis at variable scan rates revealed a thermal event at $\sim-10{ }^{\circ} \mathrm{C}$ consistent with a glass transition $\left(\mathrm{T}_{\mathrm{g}}\right)$ (Figure S14).

Simulation of a crystal lattice representative of the observed scattering data was pursued computationally using molecular dynamics (MD) simulations. Multiple (1-n'-divinyl)cyclobutane oligomers $(n=17)$ were generated with sequence distributions (syn/anti diads) consistent with those observed by NMR spectroscopy. Low energy conformers obtained from the molecular mechanics simulations were used as inputs to simulate crystalline polymorphs from which a series of low-energy $\mathrm{P} 2{ }_{1}$ symmetric unit-cells were obtained (see Supplementary Information for additional details). The polymorph ( $\mathrm{P} 2_{1} ; \mathrm{a}=29.76, \mathrm{~b}=10.45$, and $\mathrm{c}=10.53 \AA ; \alpha=90.00^{\circ}, \beta=73.64^{\circ}, \gamma=90.00^{\circ}$ ) that best reproduced the WAXS scattering data is shown in Figure 5A and 5B. The resulting strand is comprised primarily of alternating syn and anti dispositions of cyclobutyl rings, consistent with the NMR and computational data (vide supra). Construction of a supercell of energy minimized conformers resulted in the packing distribution depicted in Figure 5C, which was able to accurately reproduce the room temperature powder diffraction data. MD simulations were also used to generate an amorphous supercell generating a simulated scattering pattern consistent with experimental data obtained at $170{ }^{\circ} \mathrm{C}$ (Figure S11). 

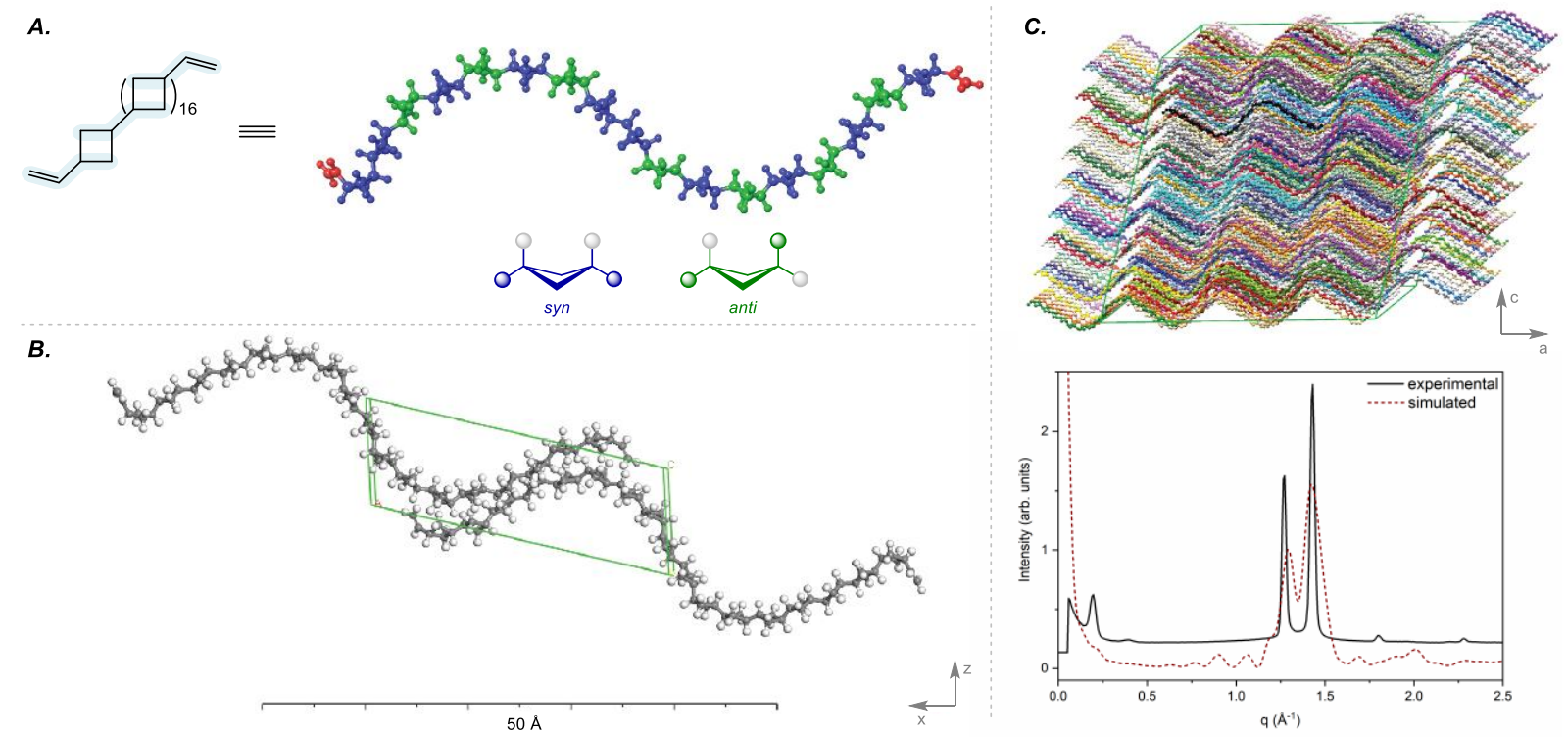

Figure 5. Molecular Dynamics simulated (1,17'-divinyl)polycyclobutane oligomer. A. Depiction of an energyminimized single strand oligomer. Vinyl end-groups are colored red, syn and anti colored diastereomers are shown in blue and green, respectively. B. Depiction of a plane of the $\mathrm{P} 2{ }_{1}$ crystal used to construct the supercell for prediction of the powder diffraction data. C. Top: Final frame of $1 \mathrm{~ns}$ MD equilibrated $\mathrm{P} 2_{1}$ supercell used for X-ray predictions, colored by individual oligomer chain. Hydrogens have been omitted for clarity. Bottom: Experimental WAXS data $\left(30^{\circ} \mathrm{C}\right)$ overlaid with simulated scattering data from the MD equilibrated supercell $\left(\mathrm{P} 2{ }_{1} ; \mathrm{a}=93.32 \AA\right.$, $\left.\mathrm{b}=88.45 \AA, \mathrm{c}=92.02 \AA ; \alpha=89.99^{\circ}, \beta=74.96^{\circ}, \gamma=89.99^{\circ}\right)$.

A key motivator for pursuing the [2+2]-cycloaddition/oligomerization strategy was the potential for reversibility and chemical recycling. While ring-opening $\mathrm{C}-\mathrm{C}$ oxidative addition of vinylcyclobutane with $\left(\left({ }^{\mathrm{Me}} \mathrm{PDI}\right) \mathrm{FeN}\right)_{2}\left(\mu-\mathrm{N}_{2}\right)$ and cycloreversion had been demonstrated previously in stoichiometric experiments ${ }^{20}$, the viability of this reverse reaction under catalytic conditions had yet to be demonstrated. The catalytic decomposition of such structures was posited to be potentially challenging given the strong energetic preference for coordination of butadiene to the iron catalyst. Control experiments indicated that sequestration of the butadiene formed upon retro-cycloaddition was key to achieving meaningful levels of deoligomerization. As such, activated $5 \AA$ molecular sieves were added to a benzene- $d_{6}$ solution containing $\left(\left({ }^{\mathrm{Me}} \mathrm{PDI}\right) \mathrm{FeN}\right)_{2}\left(\mu-\mathrm{N}_{2}\right)$ and vinylcyclobutane to sequester any liberated butadiene. After 6 days at $50{ }^{\circ} \mathrm{C}$, reisolation of the adsorbed volatiles from the sieves and ${ }^{1} \mathrm{H}$ NMR analysis established $99 \%$ consumption of the vinylcyclobutane and recovery of the constituent ethylene (75\% yield) and butadiene (94\% yield; Figure 6A). Likewise, catalytic retro-[2+2]-cycloaddition of 3-vinyl-1,1'-dicyclobutane, isolated from the [2+2] cycloaddition/oligomerization of ethylene and butadiene (see Supporting Information for details), was reverted to the starting hydrocarbons. Under the same conditions, the deoligomerization of 3-vinyl-1,1'dicyclobutane regenerated $57 \%$ of total ethylene and $99 \%$ of total butadiene (Figure 6B). 
A.
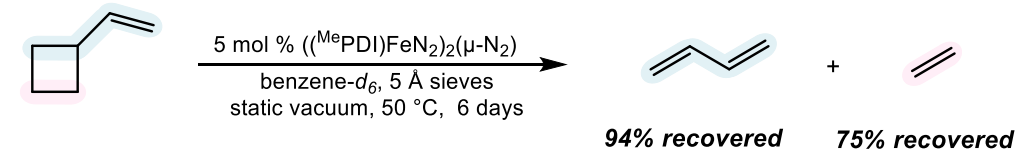

B.
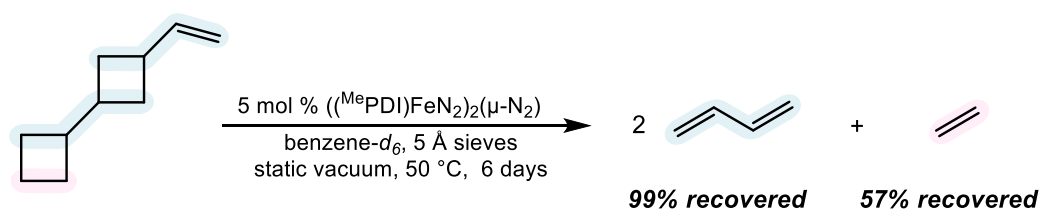

C.

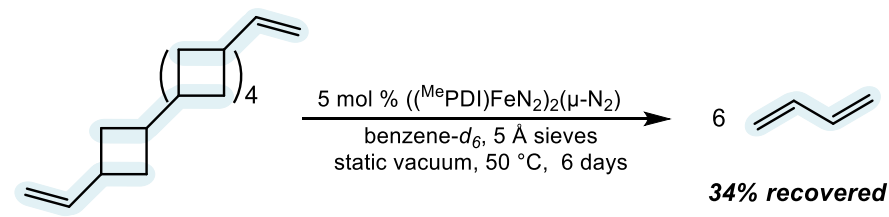

Figure 6. Catalytic chemical recycling of cyclobutane motifs. A. Depolymerization of vinylcyclobutane. B. Depolymerization of 3-vinyl-1,1'-bicyclobutane. C. Partial depolymerization of (1,5'-divinyl)polycyclobutane.

Extension to catalytic chemical recycling of (1,n'-divinyl)oligocyclobutane was demonstrated on the organic soluble material with a number-average length of 5 cyclobutyl rings $\left(\mathrm{M}_{\mathrm{n}}=324 \mathrm{~g} \mathrm{~mol}^{-1}\right)$, obtained from an oligomerization reaction run for 24 hours. In benzene- $d_{6}$ solution under static vacuum, $34 \%$ of butadiene was recovered after heating at $50{ }^{\circ} \mathrm{C}$ for 6 days (Figure 6C). The success of the deoligomerization was dependent on the volume of reaction vessel used, likely a result of product inhibition of butadiene on the activity of the catalyst. Indeed, examination of a catalytic deoligomerization by ${ }^{1} \mathrm{H}$ NMR spectroscopy indicated that butadiene coordination to the iron catalyst inhibits the retro-cycloaddition process (Figure S16). Nevertheless, the ability to recover pristine butadiene from the oligomer clearly establishes this hydrocarbon oligomer is amenable to chemical recycling using the same catalyst used for its synthesis. This is also reflected in the DFT/TST simulated free energetics, where the growing polymer chain (INT4, Figure $\mathrm{S} 17)$ and the butadiene coordinated ( $\left.{ }^{\mathrm{Me}} \mathrm{PDI}\right) \mathrm{Fe}\left(\mathrm{AD} 1\right.$, Figure S17) precatalyst are within $\sim 4 \mathrm{kcal} \mathrm{mol}^{-1}$, indicating that reversible cycloaddition/oligomerization is energetically accessible.

Conclusions. In summary, sequential [2+2]-cycloaddition of feedstock olefins mediated by pyridine(diimine)-ligated iron catalysts produced oligomeric chains consisting of cyclobutyl rings linked in the 1,3 positions. These unprecedented architectures were chemically deoligomerized back to pristine monomer and thus constitute a rare class of semi-crystalline, chemically recyclable hydrocarbon oligomers derived from inexpensive, ubiquitous feedstock olefins. Current efforts are directed toward preparation of more stereoregular (e.g. iso- and syndiotactic) material and products with higher molecular weight. 


\section{References}

1. Geyer R, Jambeck JR, Law KL. Production, use, and fate of all plastics ever made. Sci Adv 2017, 3(7): e1700782.

2. Rahimi A, García JM. Chemical recycling of waste plastics for new materials production. Nat Rev Chem 2017, 1(6): 0046.

3. Garcia JM, Robertson ML. The future of plastics recycling. Science 2017, 358(6365): 870-872.

4. Sardon H, Dove AP. Plastics recycling with a difference. Science 2018, 360(6387): 380-381.

5. Hopewell J, Dvorak R, Kosior E. Plastics recycling: challenges and opportunities. Philos Trans $R$ Soc Lond B Biol Sci 2009, 364(1526): 2115-2126.

6. Zhang X, Fevre M, Jones GO, Waymouth RM. Catalysis as an Enabling Science for Sustainable Polymers. Chem Rev 2018, 118(2): 839-885.

7. Helms BA, Russell TP. Reaction: Polymer Chemistries Enabling Cradle-to-Cradle Life Cycles for Plastics. Chem 2016, 1(6): 816-818.

8. Hong M, Chen EYX. Chemically recyclable polymers: a circular economy approach to sustainability. Green Chem 2017, 19(16): 3692-3706.

9. Rowan SJ, Cantrill SJ, Cousins GRL, Sanders JKM, Stoddart JF. Dynamic Covalent Chemistry. Angew Chem Int Ed 2002, 41(6): 898-952.

10. Liu T, Hao C, Wang L, Li Y, Liu W, Xin J, et al. Eugenol-Derived Biobased Epoxy: Shape Memory, Repairing, and Recyclability. Macromolecules 2017, 50(21): 8588-8597.

11. Ogden WA, Guan Z. Recyclable, Strong, and Highly Malleable Thermosets Based on Boroxine Networks. J Am Chem Soc, 2018, 140(20): 6217-6220.

12. Zhu J-B, Watson EM, Tang J, Chen EY-X. A synthetic polymer system with repeatable chemical recyclability. Science 2018, 360(6387): 398-403.

13. Christensen PR, Scheuermann AM, Loeffler KE, Helms BA. Closed-loop recycling of plastics enabled by dynamic covalent diketoenamine bonds. Nat Chem 2019, 11(5): 442-448.

14. Lian Z, Bhawal BN, Yu P, Morandi B. Palladium-catalyzed carbon-sulfur or carbon-phosphorus bond metathesis by reversible arylation. Science 2017, 356(6342): 1059-1063.

15. García JM. Catalyst: Design Challenges for the Future of Plastics Recycling. Chem 2016, 1(6): 813-815.

16. Feldman D. Polymer History. Des Monomers Polym 2008, 11(1): 1-15.

17. Amghizar I, Vandewalle LA, Van Geem KM, Marin GB. New Trends in Olefin Production. Eng $J$ 2017, 3(2): 171-178.

18. Pastine SJ. Reaction: Design with the End in Mind. Chem 2016, 1(6): 818-819. 
19. Long TE. Reaction: Benign by Design Demands Innovation. Chem 2017, 2(1): 7-8.

20. Russell SK, Lobkovsky E, Chirik PJ. Iron-Catalyzed Intermolecular [ $2 \pi+2 \pi]$ Cycloaddition. $J A m$ Chem Soc, 2011, 133(23): 8858-8861.

21. Hoyt JM, Schmidt VA, Tondreau AM, Chirik PJ. Iron-catalyzed intermolecular [2+2] cycloadditions of unactivated alkenes. Science 2015, 349(6251): 960-963.

22. Hoyt JM, Sylvester KT, Semproni SP, Chirik PJ. Synthesis and Electronic Structure of Bis(imino)pyridine Iron Metallacyclic Intermediates in Iron-Catalyzed Cyclization Reactions. J Am Chem Soc, 2013, 135(12): 4862-4877.

23. Schmidt VA, Hoyt JM, Margulieux GW, Chirik PJ. Cobalt-Catalyzed $[2 \pi+2 \pi]$ Cycloadditions of Alkenes: Scope, Mechanism, and Elucidation of Electronic Structure of Catalytic Intermediates. $J$ Am Chem Soc, 2015, 137(24): 7903-7914.

24. Hall Jr HK. Synthesis and polymerisation of polycyclic compounds with strained C $-\mathrm{C}$ single bonds. British Polymer Journal 1972, 4(5): 371-389.

25. Hall Jr. HK, Ykman P. Addition polymerization of cyclobutene and bicyclobutane monomers. Journal of Polymer Science: Macromolecular Reviews 1976, 11(1): 1-45.

26. Wu CC, Lenz RW. Thermal and autoxidation reactions of poly-3-methylenecyclobutene and poly1-methyl-3-methylenecyclobutene. Journal of Polymer Science Part A-1: Polymer Chemistry 1972, 10(12): 3555-3567.

27. Bandrup J, Immergut E, Grulke E. Polymer Handbook 4edn. Wiley-Blackwell: New York, 2003.

Data and Materials Availability: All data necessary to support the conclusions of this paper are available in the supplementary materials, including Materials and Methods, Figures S1 to S17, Tables S1 to S6, References S1 to S34, and optimized DFT coordinates and energetics, calculated free energies, and MD equilibrated coordinates.

Acknowledgements: We are grateful to Dr. Klemenz and the Schoop laboratory (Princeton University) for initial assistance with powder diffraction, as well as Daniel Gregory (Princeton University) for assistance with TGA/GCMS and DSC. M. M. B. thanks Kenith Conover (Princeton University) with assistance with high temperature NMR experiments. M. M. B, C. R. K. and P. J. C. thank Firmenich for initial support of this work. C.R.K. thanks the NIH for a Ruth L. Kirschstein National Research Service Award (F32 GM126640). All authors thank ExxonMobil for support of this research.

Author Contributions: C. R. K. and P. J. C. conceived the project. C. R. K. and M. M. B. performed experiments regarding the synthesis and partial characterization of oligomers. A. E. C. and S. J. M. conducted full NMR characterization and peak assignments. M. M. B. and J. A. T. performed experiments on thermal stability and crystallinity of the oligomer. J. M. Y. conducted TD/DFT and molecular mechanics calculations. M. M. B. performed the chemical recycling experiments.

Competing Interests: C. R. K. and P. J. C. are inventors on the patent application US 2019/0211142 A1 titled "Oligomeric and Polymeric Species Comprising Cyclobutane Units". J. M. Y., A. E. C., S. J. M. and J. A. T. are employees of ExxonMobil Chemical Company. 


\section{Graphical Abstract:}



